\title{
Clinicopathologic Analysis of Invasive Micropapillary Differentiation in Breast Carcinoma
}

\author{
Hind Nassar, M.D., Tracy Wallis, B.S., Aleodor Andea, M.D., Jyotirmoy Dey, Ph.D., Volkan Adsay, M.D., \\ Daniel Visscher, M.D. \\ Departments of Pathology and Biostatistics, Harper Hospital, Wayne State University and the Karmanos \\ Cancer Institute, Detroit, Michigan
}

Invasive micropapillary carcinoma (IMPCa) of breast is histologically characterized by growth of cohesive tumor cell clusters within prominent clear spaces resembling dilated angiolymphatic vessels. In this study, eighty three breast carcinomas with IMPCa differentiation were identified by review of the invasive carcinoma cases in our institution and correlated retrospectively with standard clinicopathologic parameters and survival status relative to a control series of cases (mean follow up 7 years). IMPCa growth pattern was present in $6 \%$ of all breast carcinomas; it was generally a focal component in otherwise typical invasive ductal carcinoma. It comprised more than $80 \%$ of the total neoplasm in only 10 cases $(12 \%), 50-80 \%$ of the neoplasm in 7 cases $(8 \%), 20-50 \%$ of the neoplasm in 22 cases (26\%) and less than $20 \%$ in 44 cases $(53 \%)$. The mean tumor size was $4 \mathrm{~cm}, 22 \%$ invaded skin, and $58 \%$ were poorly differentiated, but $71 \%$ were ER positive. Axillary node metastases were present in $77 \%$ of cases, were typically multiple $(51 \%$ had three or more positive), and usually contained an IMPCa component $(81 \%$ of the cases). There was no significant difference in node status, ER status, size, tumor grade, or peritumoral angiolymphatic invasion between tumors with predominant (more than $50 \%$ ) v/s focal IMPCa components. In both groups $46 \%$ of the patients died from their disease (mean interval to death $=36 \mathrm{~m})$. Skin involvement and nodal status were the only parameters which predicted poor survival $(P=.01)$. The outcome of patients with IMPCa did not differ significantly from infiltrating ductal carcinomas of similar node status. In conclusion, our results suggest that IMPCa growth pattern may be a manifestation of aggressive behavior, as shown by frequent skin invasion

Copyright (C) 2001 by The United States and Canadian Academy of Pathology, Inc.

VOL. 14, NO. 9, P. 836, 2001 Printed in the U.S.A.

Date of acceptance: May 25, 2001.

Address reprint requests to: D. Visscher, M.D., Harper Hospital, Department of Pathology, 3990 John Road, Detroit, MI 48201; e-mail: CShuch@dmc.org; fax: 313-745-9299. and extensive nodal involvement. However, clinicopathologic features and outcome of IMPCa are not strongly dependent on the relative amount of micropapillary component.

KEY WORDS: Breast carcinoma, Histology, Invasive micropapillary subtype.

Mod Pathol 2001;14(9):836-841

Histological subtype is one of the classical parameters which determine the natural history and outcome in breast cancer (1-3). Most special subtypes, including tubular, mucinous (colloid), and papillary carcinomas confer a favorable prognosis relative to infiltrating ductal carcinoma NOS (4-6). They are often localized at the time of diagnosis, and when nodes are involved, the number of metastases is usually three or less (7). Breast carcinoma subtypes are also characterized by distinctive patterns of growth and metastasis, as well as associations with germline mutations of genes such as BRCA1 and BRCA2 $(8,9)$. They are often admixed with areas of conventional infiltrating ductal carcinoma.

Invasive micropapillary carcinoma (IMPCa) is a histologic pattern of breast cancer initially described by Tavassoli and later by Luna-Moré et al. $(10,11)$. It is characterized by small, tightly cohesive groups of neoplastic cells disposed within well delineated clear spaces resembling lymphatic vessels. IMPCa has a high frequency of axillary node metastasis, present in up to $100 \%$ of the cases in some studies (11), in keeping with an angioinvasive phenotype. Thus IMPCa is thought to represent an aggressive subtype of breast carcinoma. However, series reported to date are small with short follow-up intervals. Moreover, pure invasive micropapillary growth pattern is rarely observed; most reported cases have been mixed with invasive ductal carcinoma, NOS. The clinical significance of IMPCa extent is not clear.

In this study, we retrospectively analyzed a series of breast carcinomas with variable proportions of IMPCa. Our objective was to clarify the significance 
of IMPCa phenotype by correlating the presence and degree of this pattern of differentiation with standard clinicopathologic parameters and survival status.

\section{MATERIALS AND METHODS}

We reviewed 1400 cases of invasive breast cancer occurring between 1973 and 1999 at Harper Hospital, Karmanos Cancer Institute, Detroit, Michigan. From this series, eighty three cases of invasive ductal carcinoma with variable IMPCa component were found. The specimens containing IMPCa included: core biopsy (2), excisional (open) biopsy (32), simple mastectomy (3), modified radical mastectomy (42), and radical mastectomy (4). Slides from specimens corresponding to each case, such as those from mastectomy following a core biopsy, were subsequently retrieved and reviewed. Invasive micropapillary pattern was defined as a component of neoplasm exhibiting clusters of crowded, discrete small groups of neoplastic cells arranged in solid nests or in tubules within clear spaces lined by attenuated spindle cells, suggestive of lymphatic invasion, as first described by Siriaunkgul et al. (10) (Figs. 1 and 2). Histologic parameters evaluated in each tumor included: 1) percentage of IMPCa component, based on evaluation of all the slides with semi-quantification of approximate percentages, 2) overall nuclear grade, 3) presence of angiolymphatic invasion, and 4) skin invasion. Vascular invasion was considered to be positive if tumor cell nests were present within definite vascular lumina outside of tumor, in the surrounding peritumoral stroma. Skin invasion was defined by the presence of tumor within the dermis. Presence and degree of IMPCa growth pattern were also assessed in axillary lymph node metastases. Tumor size, lymph node status, and ER status were obtained from corresponding pathology reports. Recurrence data (local

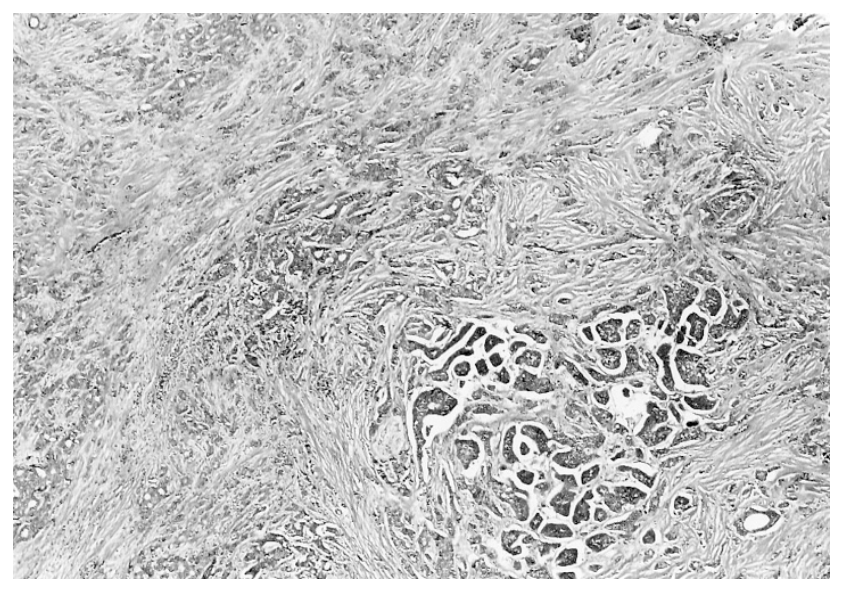

FIGURE 1. Low power view of infiltrating ductal carcinoma with focal micropapillary differentiation (right lower corner).

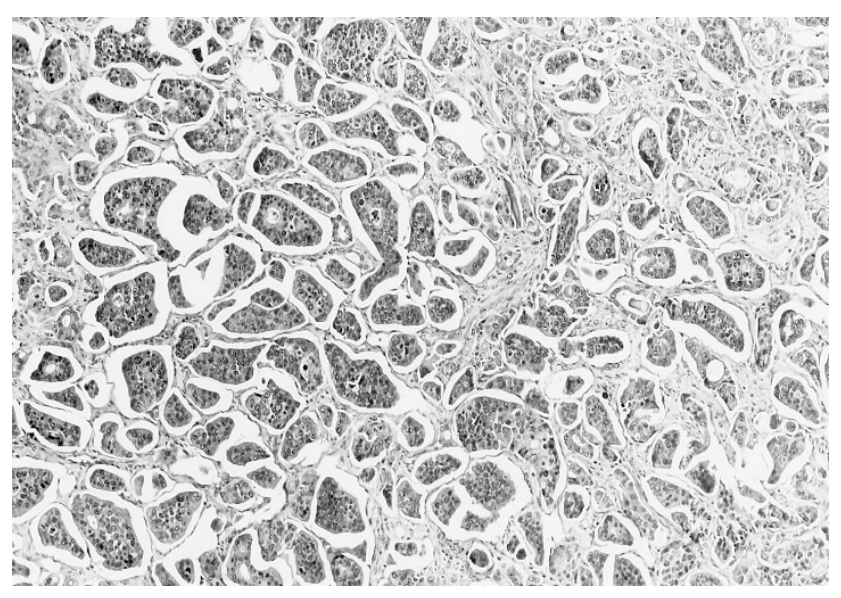

FIGURE 2. Low magnification of invasive micropapillary carcinoma (IMPCa) showing the neplastic cell clusters floating in a spongy loose stroma.

or metastasis), as well as the survival status (mean follow-up 7 years; range $=2-164 \mathrm{~m}$ ), were obtained from patient charts.

A control group of 120 stage-matched patients diagnosed with infiltrating ductal carcinoma between 1977 and 1996, inclusive, was used to analyze the survival of patients with IMPCa. Sixty-four control patients were node negative, 23 had one to three positive nodes and 33 had four or more nodes positive. Mean patients age and tumor size were similar to the IMPCa group.

An analysis of variance procedure with multiple comparisons using Bonferroni correction was employed to test equality of mean tumor size across categories defined by the percentage of IMPCa cells in the tumor. A similar procedure was followed to compare mean tumor size between alive patients, patients who died of breast cancer and those that died from other causes. For patients with positive lymph nodes were also compared across categories. Kaplan-Meier survival function estimates were obtained and log-rank tests for comparison of survival between IMPCa patients and the control group, were performed. A chi-square test for general association was used to test equality of the proportion of lymph nodes positive patients across their survival status (alive, died of disease, died of other causes). Fisher's exact test was employed to compare the same proportion across IMPCa categories in view of inadequate cell frequencies.

\section{RESULTS}

Tumors with an IMPCa component comprised $6 \%$ of the reviewed breast carcinomas. The patients were 82 females and one male ranging in age from 28 to 84 years, with a mean age of 61 years. The racial origin was known for 72 patients; 44 were 
African-American, 28 were Caucasian. Most tumors (71\%) were ER positive.

IMPCa constituted a minor proportion of the tumor volume in most cases. It comprised less than $20 \%$ of the tumor volume in 44 cases $(53 \%)$, $21-50 \%$ in 22 cases $(26 \%)$, and $51-80 \%$ in 7 cases (9\%). A diffuse micropapillary architecture ( more than $80 \%$ ) was present in only 10 cases (12\%). The dominant component in most tumors was invasive ductal carcinoma or invasive carcinoma, NOS. No other subtypes of invasive breast carcinoma were mixed with the IMPCa; in particular, no tumors with areas of mucinous (colloid) carcinoma were identified.

Areas having IMPCa growth pattern were typically identified at or near the interface of the tumor with the surrounding fibrous stroma or fatty tissue. In virtually all cases there was an abrupt transition between ductal and IMPCa patterns, easily observed at low magnification (Fig. 1). Neoplastic cells in areas of IMPCa, in general, contained more abundant cytoplasm than accompanying ductal neoplasm; the cytoplasm was also more eosinophilic in character (Fig. 3). Nuclear cytologic features were similar to the accompanying ductal component and in $57 \%$ of the cases the tumor exhibited a high overall nuclear

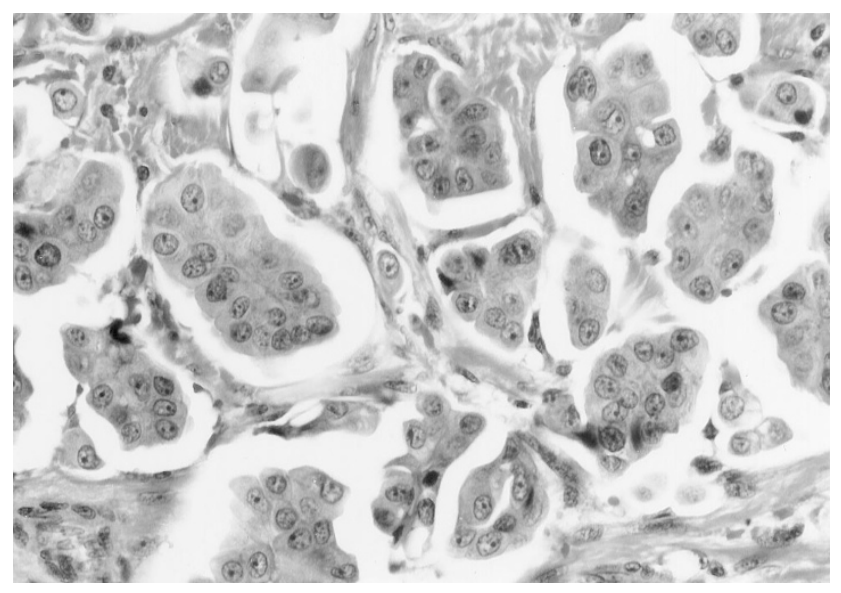

FIGURE 3. High power view of invasive micropapillary carcinoma (IMPCa). The neoplastic cells have abundant slightly granular cytoplasm with nuclear clearing. The cell clusters are surrounded by clear empty spaces grade. No correlation was found between the grade of the tumor and the proportion of IMPCa (data not shown).

Overall mean tumor size was $4.0 \mathrm{~cm}, 22 \%$ had skin involvement and in $77 \%$ of the cases, the axillary nodes were positive for metastases. Only eight patients $(9.6 \%)$ had a T1 tumor with negative nodes. Most node positive patients had multiple axillary nodal metastases; three or more lymph nodes were positive in $51 \%$ of the patients (mean number positive $=6$ ). The node metastases exhibited (at least focal) invasive micropapillary growth pattern in $81 \%$ of the involved axillary dissections. More than $50 \%$ IMPCa component was observed in $32 \%$ of the lymph node metastases (versus $21 \%$ of the primary tumors). Despite this striking degree of lymphotropism, vascular invasion, (i.e., of the conventional type) was observed in only $15 \%$ of the primary lesions overall.

A comparison between four groups of patients with different proportions of IMPCa (less than $20 \%$, $21-50 \%, 51-80 \%$, and more than $80 \%$ ) did not show any statistically significant differences, with respect to the mean age of the patients, mean tumor size, grade, ER positivity, or axillary lymph node status (Table 1).

Overall, $46 \%$ of the patients died from breast cancer with a follow-up period ranging from 2 to 143 months. Fourteen percent died from causes not related to breast cancer, mainly ischemic heart disease, sepsis, and chronic lung disease. Forty percent were alive after a follow up period ranging from 4 to 164 months (Table 2). Four patients in the alive group had local (chest wall) recurrence $(12.5 \%)$ and one has metastases to brain and bone (3.1\%). The mean follow-up period for these patients is presently 117 months.

Death due to neoplasm was not correlated with the extent of IMPCa growth. It was, however, correlated with the frequency of positive nodes (67\% had positive lymph nodes in the alive group $\mathrm{v} / \mathrm{s} 91 \%$ in the DOD group, $P=.01$ ), and with the nodal tumor burden (mean number of involved nodes $=9.2$ for DOD group versus 4.1 for survivors, $P=.01$ ). Skin invasion was strongly predictive of adverse outcome; $50 \%$ of patients

TABLE 1. Clinicopathologic Features of Invasive Micropapillary Breast Carcinoma

\begin{tabular}{|c|c|c|c|c|c|}
\hline$\%$ of IMPCa & Overall & $<20 \%$ & $21-50 \%$ & $51-80 \%$ & $>80 \%$ \\
\hline $\mathrm{N}$ & 83 & 44 & 22 & 7 & 10 \\
\hline Mean age & 61 & 61 & 63 & 61 & 53 \\
\hline Mean tumor size $(\mathrm{cm})$ & 4.0 & 4.4 & 3.1 & 4.8 & 3.1 \\
\hline Grade III & $58 \%$ & $53 \%$ & $64 \%$ & $77 \%$ & $54 \%$ \\
\hline ER positive & $71 \%$ & $74 \%$ & $75 \%$ & $60 \%$ & $62 \%$ \\
\hline $\begin{array}{l}\text { LN positive (mean \# of } \\
\text { positive LNs) }\end{array}$ & $77 \%(6)$ & $79 \%(7)$ & $70 \%(3.5)$ & $80 \%(4)$ & $100 \%(9.2)$ \\
\hline VI & $15 \%$ & $21 \%$ & $18 \%$ & $10 \%$ & $0 \%$ \\
\hline
\end{tabular}

IMPCa, invasive micropapillary carcinoma; LN, axillary lymph nodes; VI, vascular invasion. 
TABLE 2. Patient Outcome versus Clinicopathologic Features

\begin{tabular}{lclc}
\hline & DOD & Alive & Dead (other causes) \\
\hline Number of patients & $38(46 \%)$ & $32(40 \%)$ & $11(14 \%)$ \\
Mean age & 61 & 54 & 75 \\
Mean tumor size & 4.7 & 3.6 & 3.1 \\
$\quad(\mathrm{~cm})$ & & & \\
LN positive* (mean & $91 \%(9.2)$ & $67 \%(4.1)$ & $57 \%(2.7)$ \\
$\quad$ number)* & & & \\
Grade III & $57 \%$ & $56 \%$ & $63 \%$ \\
VI & $31 \%$ & $16 \%$ & $0 \%$ \\
ER positive & $67 \%$ & $79 \%$ & $57 \%$ \\
Skin invasion* & $50 \%$ & $3 \%$ & $9 \%$ \\
\% IMPCa & & & \\
$\quad<20$ & $21(52.5 \%)$ & $16(50 \%)$ & $6(54.5 \%)$ \\
$21-50$ & $10(26 \%)$ & $8(25 \%)$ & $4(36.5 \%)$ \\
51-80 & $2(7.5 \%)$ & $4(12.5 \%)$ & 0 \\
$\quad>80$ & $5(13 \%)$ & $4(12.5 \%)$ & $1(9 \%)$ \\
Mean follow-up (m) & 35.8 & 73.2 & 52.5 \\
\hline
\end{tabular}

$* \mathrm{P}<.01$.

DOD, died of disease. who died of disease had skin invasion versus only $3 \%$ of those who survived $(P<.01)$. The tumor size, histological grade, ER status, and vascular invasion were not significantly different between survivors and those who died from their disease.

Kaplan-Meier survival curve analysis showed a worse outcome overall for the IMPCa group patients relative to the control group with infiltrating ductal carcinoma (Fig. 4). However, when compared to control subsets with similar lymph node status, there was no statistically significant difference in survival between the node negative IMPCa patients, patients with 1-3 positive nodes, and patients with four or more positive nodes compared to similarly staged patients in the control group (Fig. 4).
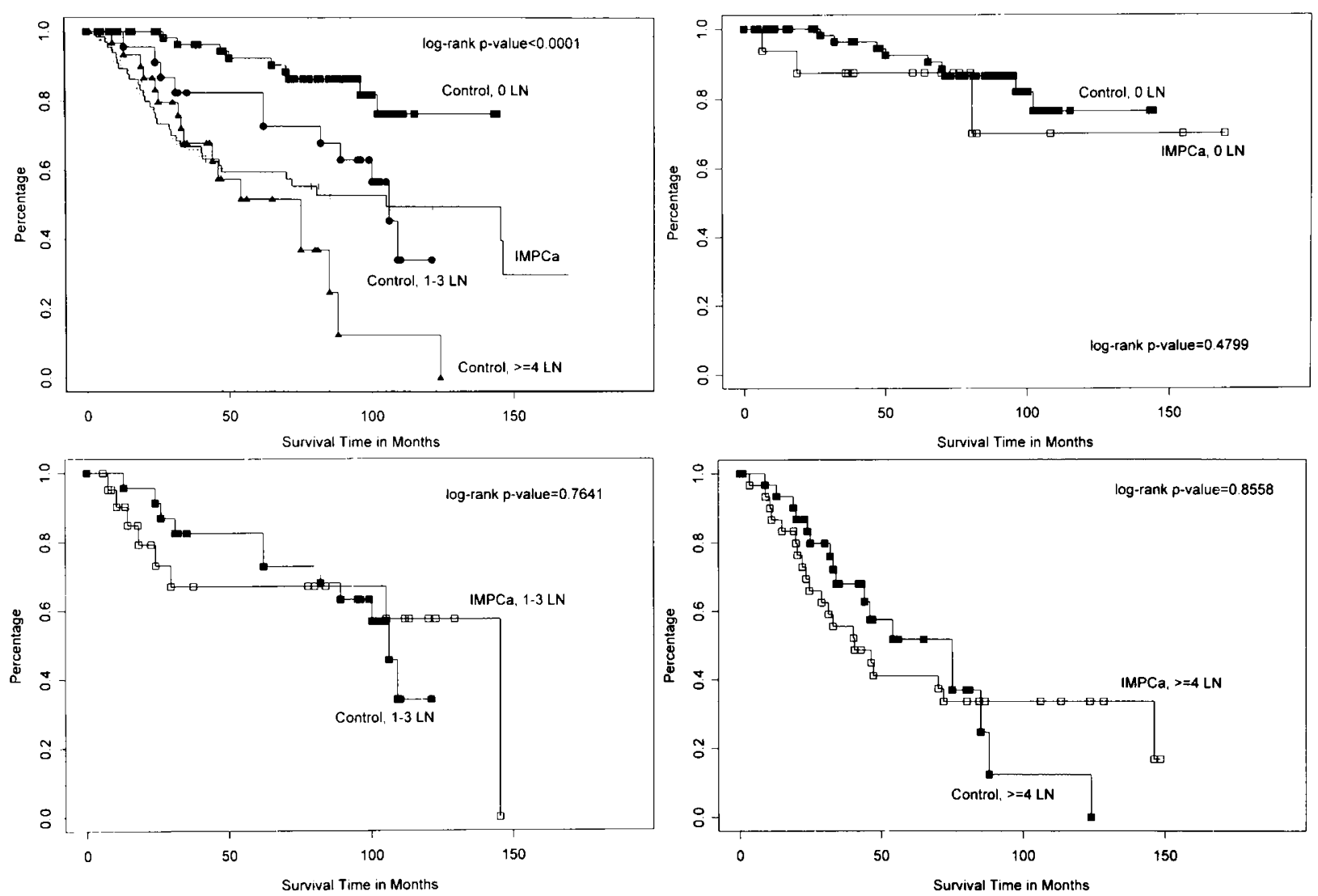

FIGURE 4. IMPCa versus Control survival as function of axillary lymph node status.

TABLE 3. Summary of Published IMPCa Series to Date

\begin{tabular}{|c|c|c|c|c|c|c|}
\hline Study (year) & $\begin{array}{c}\text { Tavassoli et al., } \\
1993(10)\end{array}$ & $\begin{array}{l}\text { Tresserra et al., } \\
1999 \text { (16) }\end{array}$ & $\begin{array}{l}\text { Middleton et al., } \\
1999 \text { (17) }\end{array}$ & $\begin{array}{c}\text { Paterakos et al., } \\
1999 \text { (13) }\end{array}$ & $\begin{array}{l}\text { Luna-More et al., } \\
2000(14)\end{array}$ & Nassar et al. \\
\hline $\mathrm{N}$ & 9 & 15 & 14 & 21 & 68 & 83 \\
\hline Mean age & 62 & 52 & 50 & 55 & 54 & 61 \\
\hline Grade III & $33 \%$ & $60 \%$ & $36 \%$ & $66 \%$ & $\sqrt{ }$ & $58 \%$ \\
\hline VI & $33 \%$ & $\mathrm{~N} / \mathrm{A}$ & $71 \%$ & N/A & 59 & $15 \%$ \\
\hline LN positive (mean \#) & $33 \%(4)$ & $60 \%(8.1)$ & $\mathrm{N} / \mathrm{A}$ & $95 \%(8.5)$ & $91 \%(9.5)$ & $77 \%(6)$ \\
\hline Size $(\mathrm{cm})$ & 1.5 & 2.3 & $3.6-6$ & 3.6 & 4.2 & 4 \\
\hline$\%$ of alive patients & $55 \%$ & $73 \%$ & $50 \%$ & N/A & $63 \%$ & $40 \%$ \\
\hline Follow-up (months) & $41-56.5$ & 43 & 54 & $\mathrm{~N} / \mathrm{A}$ & 59 & 84 \\
\hline
\end{tabular}




\section{DISCUSSION}

The results of our study show that IMPCa morphology is observed infrequently but not rarely-6\% overall-in breast unselected carcinomas. However, when present, it is usually focal. Regardless of extent, IMPCa differentiation is associated with a distinctly lymphotropic character. Despite its propensity for multiple node involvement, the outcome for IMPCa patients is not different from that of infiltrating ductal carcinoma having similar lymph node status.

Histologic features of IMPCa were documented previously $(6,10,11)$. Despite the absence of fibrovascular cores, the papillary nature of these tumors is implied by the reversed polarity of the secretory poles, confirmed by electron microscopy $(11,12)$. It is noteworthy, however that IMPCa is histologically different from conventional papillary carcinoma of the breast. The latter typically exhibits a complex arborescent growth pattern within cystically dilated duct-like spaces (6). IMPCa, on the other hand, is comprised of single, simple papillary formations, each within discrete clear spaces which are not epithelium lined. Despite the resemblance to small blood or lymphatic vessels, these spaces also lack endothelial lining and basal lamina (10).

IMPCa, in some respects, may resemble mucinous carcinoma. Although inapparent on routine $\mathrm{H} \& \mathrm{E}$ sections, the papillae are "floating" in a mucinous substance, detectable by special stains in some cases (11). However, they lack the abundant extracellular mucin seen in colloid (mucinous) carcinoma. In addition, the clusters of cells in IMPCa are typically smaller, with well defined reversed cellular polarity or tubule formation. So-called retraction artifact may also be confused with IMPCa. The former alteration, though, occurs in association with dense collagenous stroma. IMPCa, in contrast, exhibits a loose, spongy stroma.

Others have reported that breast carcinomas having IMPCa growth pattern are characterized by a remarkably high degree of regional nodal tumor spread. Luna-More et al., and Paterakos et al. reported $91 \%$ and $95 \%$ incidences of axillary node metastases respectively $(11,13)$. Involvement of multiple LNs is also typical. In our series, an average of 6 nodes were involved with axillary metastases compared to 9.5 in the study of Luna-More (14) and 8.5 in Paterakos series (13). It is possible that tumor foci with IMPCa growth pattern are a source of LN metastases, since examination of axillary contents revealed IMPCa differentiation in most cases. It may be thus hypothesized that IMPCa represents evolution of a subclone, acquiring a special morphology, and having a high propensity for regional lymphatic spread. This interpretation is further supported by the observation that foci of IMPCa growth were generally observed at the periphery (i.e., invasive growth front) of the primary tumor. The mechanism by which IMPCa facilitates nodal metastases is, at this point, unclear.

The published outcome data on IMPCa are limited (Table 3). Sixty-three percent of Luna-Moré's series of 54 patients were alive after a mean follow-up period of 59 months. The patients who died from their breast cancers had larger tumor size, higher histological grade, and a higher number of metastatic lymph nodes $(11,15)$. Paterakos et al., in their multivariate analysis of 21 cases of IMPCa, failed to confirm that IMPCa phenotype predicted more aggressive clinical behavior and a shortened disease free or overall survival (13). In our study we showed the survival of IMPCa cases was similar to that of infiltrating ductal carcinoma when matched for node status. However, the overall outcome was significantly worse for IMPCa patients. The high proportion of node positive patients with advanced metastatic tumor burden and skin involvement likely explains this difference. As such, the outcome of a patient with IMPCa will depend largely on staging parameters in a manner analogous to infiltrating ductal carcinoma.

Our data as well as those published by others, suggest that IMPCa morphology is associated with clinically meaningful staging and outcome implications. The finding that IMPCa morphology is generally microfocal, however undermines the notion of classification as distinctive histologic variant. Nevertheless, even cases with focal IMPCa growth displayed an aggressive behavior. Thus, we would propose the use of descriptive terms, such as "invasive micropapillary differentiation", in order to convey the characteristic biology of IMPCa phenotype to clinicians.

\section{REFERENCES}

1. Ellis IO, Galea M, Broughton N, Locker A, Blamey RW, Elston CW. Pathological prognostic factors in breast cancer. II. Histological type. Relationship with survival in a large study with long-term follow-up. Histopathology 1992;20:479-89.

2. Pereira H, Pinder SE, Sibbering DM, Galea MH, Elston CW, Blamey RW, et al. Pathological prognostic factors in breast cancer. IV: Should you be a typer or a grader? A comparative study of two histological prognostic features in operable breast carcinoma. Histopathology 1995;27:219-26.

3. Tabar L, Fagerberg G, Chen HH, Duffy SW, Gad A. Tumour development, histology and grade of breast cancers: prognosis and progression. Int J Cancer 1996;66:413-9.

4. Carstens PHB, Greenberg RA, Francis D, Lyon H. Tubular carcinoma of the breast. A long term follow-up. Histopathology 1985;9:271-80.

5. Clayton F. Pure mucinous carcinomas of breast: morphologic features and prognostic correlates. Hum Pathol 1986; 17:34-8.

6. Fisher ER, Palekar AS, Redmond C, Barton B, Fisher B. Pathologic findings from the National Surgical Adjuvant Breast Project (protocol no. 4) VI. Invasive papillary cancer. Am J Clin Pathol 1980;73:313-22. 
7. Donegan WL. Tumor-related prognostic factors for breast cancer. CA Cancer J Clin 1997;47:28-51.

8. Breast Cancer Linkage Consortium. Pathology of familial breast cancer: differences between breast cancers in carriers of BRCA1 or BRCA2 mutations and sporadic cases. Lancet 1997;349:1505-10.

9. Marcus JN, Watson P, Page DL, Narod ST, Lenoir GM, Tonin P, et al. Hereditary breast cancer: pathobiology, prognosis, and BRCA1 and BRCA2 gene linkage. Cancer 1996;77:697-709.

10. Siriaunkgul S, Tavassoli FA. Invasive micropapillary carcinoma of the breast. Mod Pathol 1993;6:660-2.

11. Luna-More S, Gonzalez B, Acedo C, Rodrigo I, Luna C. Invasive micropapillary carcinoma of the breast. A new special type of invasive mammary carcinoma. Pathol Res Pract 1994; 190:668-74.

12. Peterson JL. Breast carcinomas with unexpected inside out growth pattern. Rotation of polarization associated with angioinvasion [abstract]. Path Res Pract 1993;189: 780A.
13. Paterakos M, Watkin WG, Edgerton SM, Moore DH, Thor AD. Invasive micropapillary carcinoma of the breast: a prognostic study. Hum Pathol 1999;30:1459-63.

14. Luna-More S, Casquero S, Perez-Mellado A, Rius F, Weill B, Gornemann I. Importance of estrogen receptors for the behavior of invasive micropapillary carcinoma of the breast. Review of 68 cases with follow-up of 54. Pathol Res Pract 2000;196:35-9.

15. Luna-More S, de los Santos F, Breton JJ, Canadas MA. Estrogen and progesterone receptors, c-erbB-2, p53, and Bcl-2 in thirty-three invasive micropapillary breast carcinomas. Pathol Res Pract 1996;92:27-32.

16. Tresserra F, Grases PJ, Fabregas R, Fernandez-Cid A, Dexeus $\mathrm{S}$. Invasive micropapillary carcinoma. Distinct features of a poorly recognized variant of breast carcinoma. Eur J Gynaecol Oncol 1999;20:205-8.

17. Middleton LP, Tressera F, Sobel ME, Bryant BR, Alburquerque A, Grases $\mathrm{P}$, et al. Infiltrating micropapillary carcinoma of the breast. Mod Pathol 1999;12:499-504. 